\title{
Orexin Signaling in the Ventral Tegmental Area Is Required for High-Fat Appetite Induced by Opioid Stimulation of the Nucleus Accumbens
}

\author{
Huiyuan Zheng, Laurel M. Patterson, and Hans-Rudolf Berthoud \\ Neurobiology of Nutrition Laboratory, Pennington Biomedical Research Center, Louisiana State University System, Baton Rouge, Louisiana 70808
}

\begin{abstract}
The overriding of satiety and homeostatic control mechanisms by cognitive, rewarding, and emotional aspects of palatable foods may contribute to the evolving obesity crisis, but little is known about neural pathways and mechanisms responsible for crosstalk between the "cognitive" and "metabolic" brain in the control of appetite. Here we show that neural connections between the nucleus accumbens and hypothalamus might be part of this link. Using the well known model of selective stimulation of high-fat intake induced by intraaccumbens injection of the $\mu$-opioid receptor agonist D-Ala2- $N$-Me-Phe ${ }^{4}$-gly ${ }^{5}$-ol-enkephalin (DAMGO), we demonstrate that orexin signaling in the ventral tegmental area is important for this reward-driven appetite to override metabolic repletion signals in presatiated rats. We further show that accumbens DAMGO in the absence of food selectively increases the proportion of orexin neurons expressing c-Fos in parts of the perifornical hypothalamus and that neural projections originating in DAMGO-responsive sites of the nucleus accumbens make close anatomical contacts with hypothalamic orexin neurons. These findings suggest that direct accumbens-hypothalamic projections can stimulate hypothalamic orexin neurons, which in turn through orexin-1 receptor signaling in the ventral tegmental area and possibly other sites interfaces with the motivational and motor systems to increase intake of palatable food.
\end{abstract}

Key words: food intake; palatable food; high-fat diet; limbic system; nucleus accumbens; orexin-1 receptor

\section{Introduction}

Besides metabolic need, palatability and reward are thought to be major determinants for ingestion of a particular food item. Highly palatable foods, if easily available, are ingested even in a metabolically replete, satiated state. This overriding of homeostatic control mechanisms by the cognitive and rewarding aspects of food may contribute to the evolving obesity crisis (Berthoud, 2004; Volkow and Wise, 2005). The neural mechanisms of food reward are thought to be organized mainly within corticolimbic structures such as prefrontal cortex (PFC), amygdala, ventral striatum, and hypothalamus (Berridge, 1996; Kelley and Berridge, 2002; Smith and Berridge, 2005). The ventral striatum is considered as an important interface between the reward system and the motor system (Kelley, 2004); it has been said to translate motivation into action (Mogenson et al., 1980). Here we focus on the robust feeding of high-fat diet induced by administration of the $\mu$-opioid receptor agonist D-Ala2- $\mathrm{N}$-Me-Phe ${ }^{4}$-gly ${ }^{5}$-ol-enkephalin (DAMGO) to the nucleus accumbens as a model of satiety-resistant eating of palatable food (Zhang et al., 1998; Zhang and Kelley, 2000; Will et al., 2003; Pecina and Berridge, 2005).

Received Feb. 13, 2006; revised Aug. 25, 2007; accepted Aug. 25, 2007.

This work was supported by National Institutes of Health Grants DK47348 and DK071082. We thank Dr. Neil Upton and GlaxoSmithKline for the gift of the orexin blocker SB334867.

Correspondence should be addressed to Hans-Rudolf Berthoud, Pennington Biomedical Research Center, 6400 Perkins Road, Baton Rouge, LA 70808. E-mail: berthohr@pbrc.edu.

D01:10.1523/JNEUROSCI.3542-07.2007

Copyright $\odot 2007$ Society for Neuroscience $\quad 0270-6474 / 07 / 2711075-08 \$ 15.00 / 0$
Inhibiting activity in the shell of the nucleus accumbens by means of the $\mathrm{GABA}_{\mathrm{A}}$ agonist muscimol stimulates chow intake (Stratford and Kelley, 1997), and injection of the $\mu$-opioid agonist DAMGO into the nucleus accumbens selectively stimulates appetite for high-fat food (Zhang et al., 1998; Zhang and Kelley, 2000; Will et al., 2003). In either case, the feeding effects can be blocked by inhibiting neural activity in the lateral hypothalamus (LH) (Stratford and Kelley, 1999; Will et al., 2003). Projections from the nucleus accumbens, particularly the shell, to the lateral hypothalamus that could potentially play a role in accumbensinduced appetites have been described (Conrad and Pfaff, 1976; Groenewegen and Russchen, 1984; Usuda et al., 1998; Otake and Nakamura, 2000). Neurons expressing the orexigenic peptides orexin and melanin-concentrating hormone $(\mathrm{MCH})$ are well known to populate the lateral aspects of the hypothalamus (Date et al., 1999), and it has been shown that orexin injection into the third ventricle of rats induces a specific appetite for high-fat food (Clegg et al., 2002). Furthermore, orexin neurons in the lateral hypothalamus play a role in reward seeking (Harris et al., 2005), and orexin signaling in the ventral tegmental area (VTA) is involved in drug-induced plasticity and activation of the mesolimbic dopamine system (Borgland et al., 2006; Narita et al., 2006). Thus, we hypothesized that the high-fat appetite induced by DAMGO administration into the nucleus accumbens is partially mediated by activation of hypothalamic orexin neurons and their downstream signaling through the orexin receptor. We used two approaches to test this hypothesis by either blocking the orexin receptor pharmacologically or using an orexin-deficient mouse 
model. In addition, we tested the ability of accumbens DAMGO to activate hypothalamic orexin neurons using c-Fos as a marker, and we identified accumbens-hypothalamic projections using anterograde tracing from behaviorally characterized injection sites.

\section{Materials and Methods}

Animals and housing. Adult male Sprague Dawley rats (Harlan, Indianapolis, IN) weighing $310-320 \mathrm{~g}$ at the time of surgery were housed individually in hanging wire mesh cages in a climate-controlled room $\left(22 \pm 2^{\circ} \mathrm{C}\right)$ on a $12 \mathrm{~h}$ light/dark cycle with lights on at 7:00 A.M. and lights off at 7:00 P.M. Homozygous prepro-orexin knock-out mice (obtained from Dr. R. Palmiter, University of Washington, Seattle, WA) were bred at the Pennington Center. C57BL/6J wild-type mice were obtained from The Jackson Laboratory (Bar Harbor, ME). Mice were kept individually in shoebox cages. Food and water were available ad libitum except as specified below. All experimental protocols were approved by the Institutional Animal Care and Use Committee (Pennington Biomedical Research Center) and were conducted in compliance with United States Department of Agriculture regulations and American Physiological Society principles for research involving animals.

Implantation of chronic intracerebroventricular cannulas and telemetry transponders. Rats were anesthetized with ketamine/acepromazine/xylazine $(80 / 1.6 / 5.4 \mathrm{mg} / \mathrm{kg}$, s.c. $)$ and given atropine $(1 \mathrm{mg} / \mathrm{kg}$, i.p.). Bilateral thin-walled stainless steel guide cannulas ( 24 or 25 gauge) were aimed at the border between shell and core of the nucleus accumbens [anteroposterior (AP), $1.4 \mathrm{~mm}$; lateromedial (LM), $1.8 \mathrm{~mm}$; dorsoventral (DV), $-5.3 \mathrm{~mm}$ ], the ventral tegmental area (AP, $-5.0 \mathrm{~mm}$; LM, $0.9 \mathrm{~mm}$; DV, $-5.7 \mathrm{~mm}$ ), arcuate nucleus (AP, $-3.4 \mathrm{~mm}$; LM, $0.5 \mathrm{~mm}$; DV , $-8.8 \mathrm{~mm}$ ), or the paraventricular nucleus of the thalamus (PVT) (AP, $-3.4 \mathrm{~mm}$; $\mathrm{LM}, 0.5 \mathrm{~mm}$; DV,$-5.0 \mathrm{~mm}$ ). Twelve to $15 \mathrm{~d}$ were allowed for recovery from the surgery, at which time animals were given one saline injection with 31 gauge injectors extending 1.5-2.5 $\mathrm{mm}$ (depending on experiments) beyond the tip of guide cannulas for intraparenchymal injections and $0.6 \mathrm{~mm}$ for lateral ventricle injections to avoid potential side effects of initial penetration to the injection site on the behavioral response.

For mice, guide cannulas made of 26 gauge stainless steel tubing were aimed unilaterally at the nucleus accumbens (AP, $-1.1 \mathrm{~mm}$; LM, 1.2 $\mathrm{mm}$; DV,$-3.6 \mathrm{~mm}$ ). Injectors, made of 33 gauge stainless steel tubing to extend the guide cannulas by $1 \mathrm{~mm}$, were used for injections. In some mice, telemetry transponders (VitalView Series 4000; Mini Mitter, Bend, OR) were implanted into the abdominal cavity to monitor locomotor activity.

Experimental protocol and measurement of food intake. Rats maintained on regular lab chow were trained to eat high-fat diet (60\% energy from fat, D12492; Research Diets, New Brunswick, NJ) for 2 h every other day between 9:00 A.M. and 11:00 A.M. during recovery from surgery. On experimental days, chow was replaced with high-fat diet at 9:00 A.M., and $1 \mathrm{~h}$ intake was measured in all but the VTA cannulated group. Because of unusually high baseline high-fat intake, VTA cannulated animals were given overnight high-fat diet the night before experimental days to achieve comparable baselines with other injection sites. After this presatiation period, rats were pretreated by injecting the orexin-1 receptor (OX1R) antagonist SB334867 [1-(2-methylbenzoxazol-6-yl)-3$[1,5]$ naphthyridin-4-yl-urea hydrochloride] (GlaxoSmithKline, London, UK) into the lateral ventricle $(30,60$, or $100 \mathrm{nmol}, 3.0 \mu \mathrm{l})$, the ventral tegmental area $(15 \mathrm{nmol} / 0.5 \mu \mathrm{l}$ per side), the arcuate nucleus (15 $\mathrm{nmol} / 0.3 \mu \mathrm{l}$ per side), or the paraventricular nucleus of the thalamus ( 15 $\mathrm{nmol} / 0.3 \mu \mathrm{l}$ per side), in separate groups of rats. The antagonist was dissolved in $10 \%$ hydroxypropyl- $\beta$-cyclodextrin (Fisher, Pittsburgh, PA), which also served as a vehicle. Twenty minutes later, either DAMGO (250 ng in $0.5 \mu \mathrm{l}$ of saline; Sigma, St. Louis, MO) or saline alone was injected into the nucleus accumbens. Injections were made over a period of $1 \mathrm{~min}$, and the injector was left in place for 1 additional minute to prevent backflow. Immediately after injections, rats were put back into their home cage, and intake of high-fat diet was measured for 1-2 h. To prevent receptor desensitization with repeated DAMGO injections, we minimized the exposure to DAMGO to a given injection site by injecting it unilaterally and alternating between left and right accumbens. Pilot experiments showed that unilateral and bilateral injection of DAMGO produced similar effects.

Mice maintained on regular mouse chow were trained to drink $20 \%$ corn oil emulsion (commercially available corn oil emulsified in water with $0.25 \%$ Emplex and $0.175 \%$ xanthan gum) from a sipper tube in their home cage during several days of the recovery period. On test days, chow was removed, and, $1 \mathrm{~h}$ later, either DAMGO (20 and $35 \mathrm{pmol}$ in $200 \mathrm{nl}$ of saline) or saline alone was injected into the nucleus accumbens. Thirty minutes after the injection, they were given access to $20 \%$ corn oil emulsion, and intake was continuously monitored for $2 \mathrm{~h}$ using a lick sensor system (VitalView Data Acquisition System Series 4000; Mini Mitter).

Stimulation of c-Fos. A separate group of naive rats with bilateral cannulas aimed at the nucleus accumbens was used for DAMGO-induced c-Fos induction. Rats were adapted to the injection procedure as usual. On test days, food was removed, and, $1 \mathrm{~h}$ later, either DAMGO (250 ng in $500 \mathrm{nl}$ of saline) or saline alone was injected bilaterally into the nucleus accumbens. After injections, rats were returned to their home cage without access to food and, after $90 \mathrm{~min}$, were killed and transcardially perfused.

Anterograde tracing with biotinylated dextran amine. A few rats used in the orexin receptor blocking experiments above were also used for biotinylated dextran amine (BDA) anterograde tracing. Using the same presatiation protocol, DAMGO (250 ng) and the anterograde tracer biotinylated dextran amine (molecular weight of 10,000, 9\%; Invitrogen, Carlsbad, CA) were combined in the same injection volume of $0.5 \mu \mathrm{l}$ of sterile saline. Pilot experiments showed that presence of BDA in the injection site did not change the behavioral response to DAMGO. At the end of the behavioral experiments, rats were killed and transcardially perfused for verification of the injection site and immunohistochemical processing of BDA.

Tissue processing and immunohistochemistry. Rats and mice were deeply anesthetized with pentobarbital sodium $(90 \mathrm{mg} / \mathrm{kg}$ ) and transcardially perfused with heparinized saline $(20 \mathrm{U} / \mathrm{ml})$, followed by ice cold, $4 \%$ phosphate-buffered ( $\mathrm{pH}$ 7.4) paraformaldehyde. Brains were extracted, blocked, and postfixed in the same fixative overnight. Before cryosectioning, the tissue was immersed for $24 \mathrm{~h}$ in an $18 \%$ sucrose solution in PBS with $0.05 \%$ sodium azide. Free-floating, frozen sections of $30 \mu \mathrm{m}$ were cut, separated into five series, and either processed immediately or stored in cryoprotectant solution at $-20^{\circ} \mathrm{C}$.

A complete series (20-25 sections) of one-in-five sections stretching the entire rostrocaudal dimension of the hypothalamus was processed for c-Fos immunohistochemistry using the $\mathrm{ABC}$ /nickel-enhanced 3,3' diaminobenzidine tetrahydrochloride (NiDAB) method. Briefly, the tissue was pretreated with a solution of $0.5 \%$ sodium borohydride in PBS. Appropriate washes in PBS followed this and subsequent incubations. For quenching endogenous peroxidase, sections were treated with $3 \%$ hydrogen peroxide/methanol (1:4) before blocking for $2 \mathrm{~h}$ in a solution of PBS with $0.5 \%$ Triton X-100 (PBST) containing 5\% normal goat serum and $1 \%$ bovine serum albumin. Incubation in the primary antibody (c-Fos AB-5, 1:30,000; Oncogene/EMD Biosciences, La Jolla, CA) was for $20 \mathrm{~h}$ at room temperature (RT) and was followed by $2 \mathrm{~h}$ in biotinylated goat anti-rabbit secondary antibody (1:500; Jackson ImmunoResearch, West Grove, PA). The sections were then incubated for $1 \mathrm{~h}$ in avidin-biotin complex (1:500; Vectastain ABC Elite kit; Vector Laboratories, Burlingame, CA). The blue-black nuclear Fos was visualized using a NiDAB substrate kit (Pierce, Rockford, IL).

Fos staining was followed by immunofluorescent labeling for orexin-A (ORX). Sections were reincubated with the blocking solution before incubation in the second primary antiserum, rabbit anti-ORX (1:100; Oncogene/EMD Biosciences) for $20 \mathrm{~h}$ at RT or $40 \mathrm{~h}$ at $7^{\circ} \mathrm{C}$. The secondary antibody, Alexa 594 goat anti-rabbit IgG (1:2000; Invitrogen), was applied for $2 \mathrm{~h}$ at RT in the dark. After $1 \mathrm{~h}$ in $70 \%$ glycerol, the sections were mounted in $100 \%$ glycerol with the anti-fade agent $5 \% n$-propyl gallate.

Double labeling for BDA and ORX was done simultaneously. After sodium borohydride treatment, sections were blocked in 5\% donkey serum in PBST before incubation in the two primary antibodies, mouse anti-biotin (1:1000; Sigma) and goat anti-orexin-A (1:8000; Santa Cruz Biotechnology, Santa Cruz, CA). For secondary labeling, fluorescent 
conjugates of donkey IgG (Jackson ImmunoResearch), anti-mouse cyanine 3 (Cy3) (1:600, for biotin) and anti-goat Cy2 (1:200, for ORX), were diluted in PBST, and the tissue was incubated and mounted as above.

Staining was absent in control experiments with omission of the primary antibodies or with incubation in primary antibody that had been preadsorbed with the respective peptide.

Counting procedures, imaging, and statistical analysis. For the quantitative assessment of Fos expression in the lateral hypothalamus, three to five sections from -2.5 to $-3.8 \mathrm{~mm}$ (from bregma) were selected, and images were generated through a $20 \times$ objective with a confocal microscope. The rectangular field captured was chosen for each side of each section to include an extended perifornical area as shown in Figure 6. Fos-positive cell nuclei were visualized using the $633 \mathrm{~nm}$ line of a helium/ neon laser in the transmitted light mode and stored in one channel (green). Red fluorescent peptide immunoreactivity within a $10 \mu \mathrm{m}$ slice (centered around the plane used for the Fos image) was captured in the confocal mode using the $568 \mathrm{~nm}$ line of an argon/krypton laser by collapsing 10 optical sections, $1 \mu \mathrm{m}$ apart into one horizontal plane and stored in a second channel (red).

Images were then displayed on a full screen using imaging software that allowed tagging each individual neuron (Image-Pro Plus; Media Cybernetics, Silver Spring, MD). Neurons were classified as Fos only, peptide only, or double labeled according to the presence above background of black $\mathrm{NiDAB}$ (Fos) reaction product in the cell nucleus and/or red peptide fluorescence in the cytoplasm.

Statistical analysis. High-fat intake data for rats were analyzed with a one-way ANOVA, followed by Tukey's multiple comparisons tests. Corn oil intake and locomotor activity were analyzed by separate two-way ANOVAs for the two time periods and followed up by planned $t$ tests. Fos, orexin, and double-labeled cell counts were analyzed by independent $t$ tests. Significance level was set at $p<0.05$.

\section{Results \\ Orexin receptor antagonist SB334867 suppresses DAMGO- induced high-fat intake in rats}

As has been shown previously (Kelley et al., 2002; Will et al., 2003), DAMGO injected into the nucleus accumbens induces robust intake of high-fat diet in the rat. In our modified paradigm, rats maintained on chow diet were presatiated by $1 \mathrm{~h}$ access to the high-fat diet using the palatability contrast to drive intake. During this hour, rats ate an average of $9.8 \pm 0.5 \mathrm{~g}(\sim 51 \mathrm{kcal})$ of the high-fat diet, representing more than half their average daily energy intake. When 15 min later saline was injected into the nucleus accumbens, rats ingested only a small additional amount of $2.5 \pm 0.6 \mathrm{~g}$ high-fat diet in another $2 \mathrm{~h}$ (Fig. 1). However, when DAMGO (250 ng/rat) was injected, rats ingested another $8.2 \pm$ $0.5 \mathrm{~g}(\sim 43 \mathrm{kcal})$ of high-fat diet $(p<0.001$ compared with saline), completely disregarding satiety signals. Previous treatment with the orexin-1 receptor antagonist SB334867, injected into the lateral ventricle, dose dependently attenuated this DAMGO-induced high-fat intake. At a dose of $60 \mathrm{nmol}$, the antagonist suppressed DAMGO-induced intake significantly to $3.4 \pm 0.9 \mathrm{~g}(-69 \% ; p<0.001)$. The highest dose of $100 \mathrm{nmol}$ used produced a similar suppression of DAMGO-induced highfat intake to $3.5 \pm 0.6 \mathrm{~g}(-70 \% ; p<0.001)$, suggesting that maximal suppression was reached and that a small part of the DAMGO effect was not dependent on orexin signaling. All injection sites were located within the core of the nucleus accumbens (Fig. 2).

In an effort to identify the critical site(s) for orexin downstream signaling, we injected the OX1R-antagonist bilaterally into several areas shown to receive heavy orexin projections. Injection of the blocker into the ventral tegmental area, an area receiving robust orexin projections (Fadel and Deutch, 2002) and involved in reward-driven food intake (Borgland et al., 2006; Harris and Aston-Jones, 2006; Narita et al., 2006),

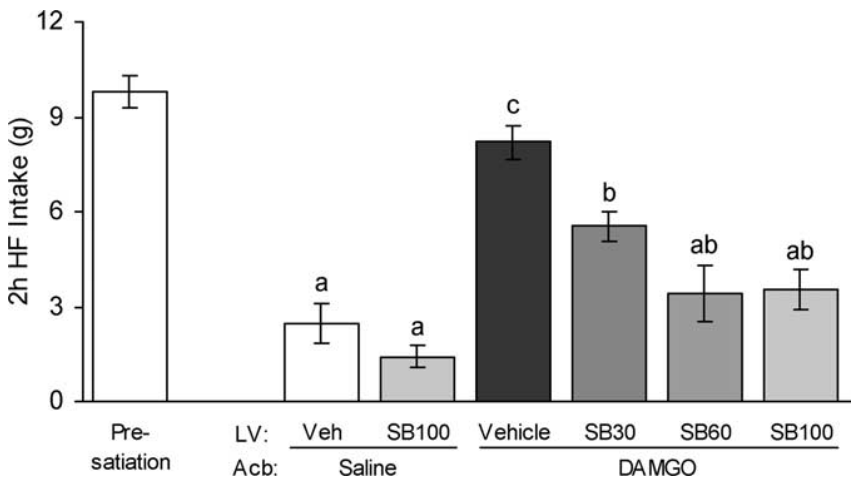

Figure 1. Orexin-1 receptor antagonist administration into the lateral ventricle attenuates high-fat intake induced by intra-accumbens injection of DAMGO in presatiated rats. Rats maintained on chow diet in were given $1 \mathrm{~h}$ access to high-fat diet. Immediately thereafter, they received either vehicle (Veh) or SB334867 (SB; 30, 60, or $100 \mathrm{nmol}$ ) injections into the right lateral ventricle (LV) and either saline or DAMGO $(250 \mathrm{ng})$ injections into the nucleus accumbens (Acb). Intake of high-fat (HF) diet was measured for $2 \mathrm{~h}$ after accumbens injections. Bars that do not share the same letter are significantly different from each other (based on ANOVA, followed by Bonferroni's adjusted multiple comparisons test, $p<0.05$ ).

almost completely blocked high-fat eating induced by accumbens DAMGO injections (Fig. 3). If the antagonist was injected outside the medial area of the VTA or when injected unilaterally, it was unable to block DAMGO-induced high-fat eating (Fig. 3)

Both NPY and pro-opiomelanocortin neurons in the arcuate nucleus also receive orexin projections, and orexin modulates their electrical activity (Guan et al., 2001; Backberg et al., 2002; Muroya et al., 2004). Furthermore, we found in a previous study that injection of the GABA agonist muscimol into the nucleus accumbens shell induced c-Fos in arcuate nucleus NPY neurons (Zheng et al., 2003), raising the possibility that orexin might be involved. However, in contrast to the VTA, SB334867 injected into the arcuate nucleus did not inhibit DAMGO-induced high-fat intake (Fig. 4a). The PVT receives a very dense orexin projection and might be an important relay in a circuit modulating feeding behavior through cholinergic striatal interneurons (Kelley et al., 2005; Parsons et al., 2006). However, bilateral SB334867 injection into the PVT did not block the DAMGO effect either (Fig. 4b). Finally, the paraventricular nucleus of the hypothalamus is also heavily innervated by orexin fibers and strongly implicated in melanocortin-4 receptor-mediated modulation of food intake (Balthasar et al., 2005). In a pilot experiment with fewer animals, we injected SB334867 (15 nmol/0.5 $\mu \mathrm{l}$ per side) into the paraventricular nucleus, but it was without effect on DAMGO-induced high-fat feeding (data not shown).

\section{Wild-type but not orexin-deficient mice exhibit DAMGO- induced increase in corn oil consumption}

To further test the role of orexin, we investigated the effect of accumbens DAMGO injections in orexin-deficient and wildtype mice trained to lick $20 \%$ corn oil emulsion from a spout. As shown in Figure $5 c$, the injection sites were confined to an area including the lateral part of the shell and medial part of the core of the nucleus accumbens. Unlike rats, both wild-type and knock-out mice were hyperactive during the first hour after injection of DAMGO into the nucleus accumbens (Fig. $5 b$ ), and this hyperactivity completely masked any effects on corn oil intake. However, during the second hour of access, accumbens DAMGO dose dependently enhanced corn oil lick- 


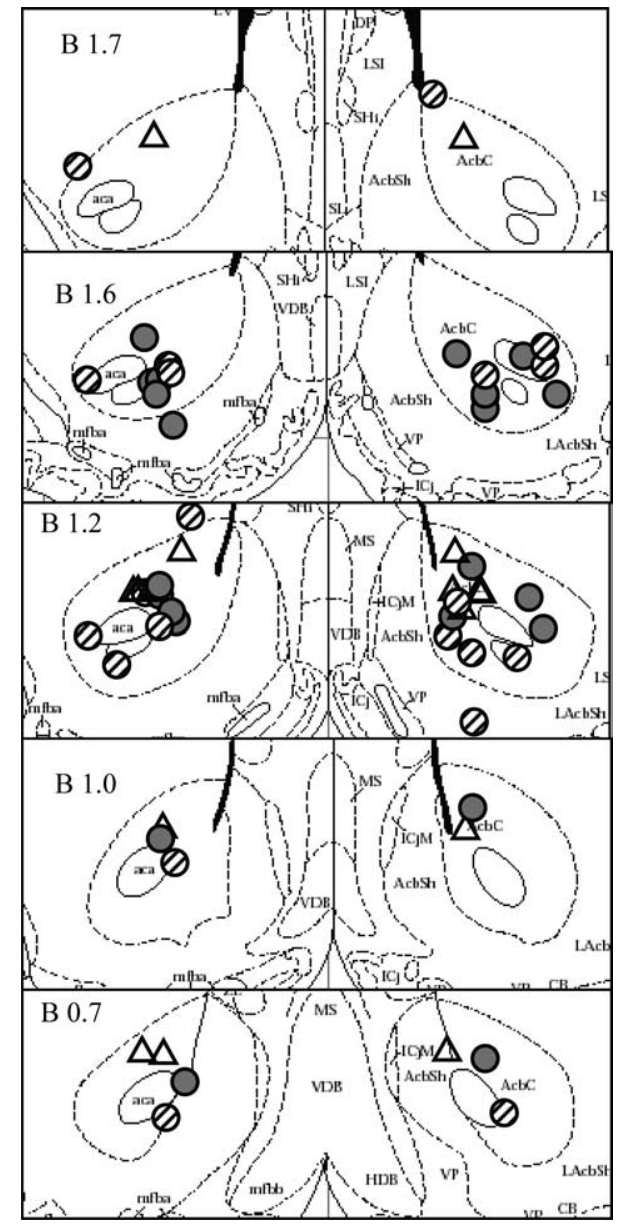

Figure 2. DAMG0 injection sites in the nucleus accumbens. Location of injection cannula tips in the nucleus accumbens of rats used for experiments with lateral ventricle administration (open triangles) and for experiments with intra-VTA administration (in VTA, striped circles; outside VTA, gray circles) of the orexin receptor antagonist SB334867. Injection sites are superimposed on images from the Paxinas and Watson (1997) stereotaxic atlas.

ing in wild-type $(p<0.05)$ but not orexin-deficient (Fig. $5 a)$ mice. Although this differential effect was in part attributable to a significantly higher baseline intake of the mutant mice $\left(t_{(9)}=3.14 ; p=0.012\right)$, the number of licks after the higher dose of DAMGO was significantly lower in knock-out compared with wild-type mice $\left(t_{(9)}=2.30 ; p=0.047\right)$. Also, during the second hour after injection, DAMGO-induced hyperactivity had almost completely disappeared, and there was no significant difference in the activity level between genotypes, making it unlikely that increased locomotor activity prevented the knock-out mice from licking (Fig. 5b).

\section{Accumbens DAMGO increases c-Fos expression in perifornical orexin neurons}

To evaluate the potential of accumbens DAMGO injections to activate hypothalamic orexin neurons, we examined c-Fos expression in the hypothalamic orexin field after injection of DAMGO into accumbens sites that yielded robust high-fat feeding responses. As shown previously (Kelley et al., 2002), accumbens DAMGO injections increased the number of neurons expressing c-Fos in certain areas of the hypothalamus (Fig. 6). DAMGO-induced Fos expression was particularly strong in an area dorsomedial to the fornix $\left(t_{(5)}=3.74 ; p=0.013\right.$ ) (Figs. 6, 7).
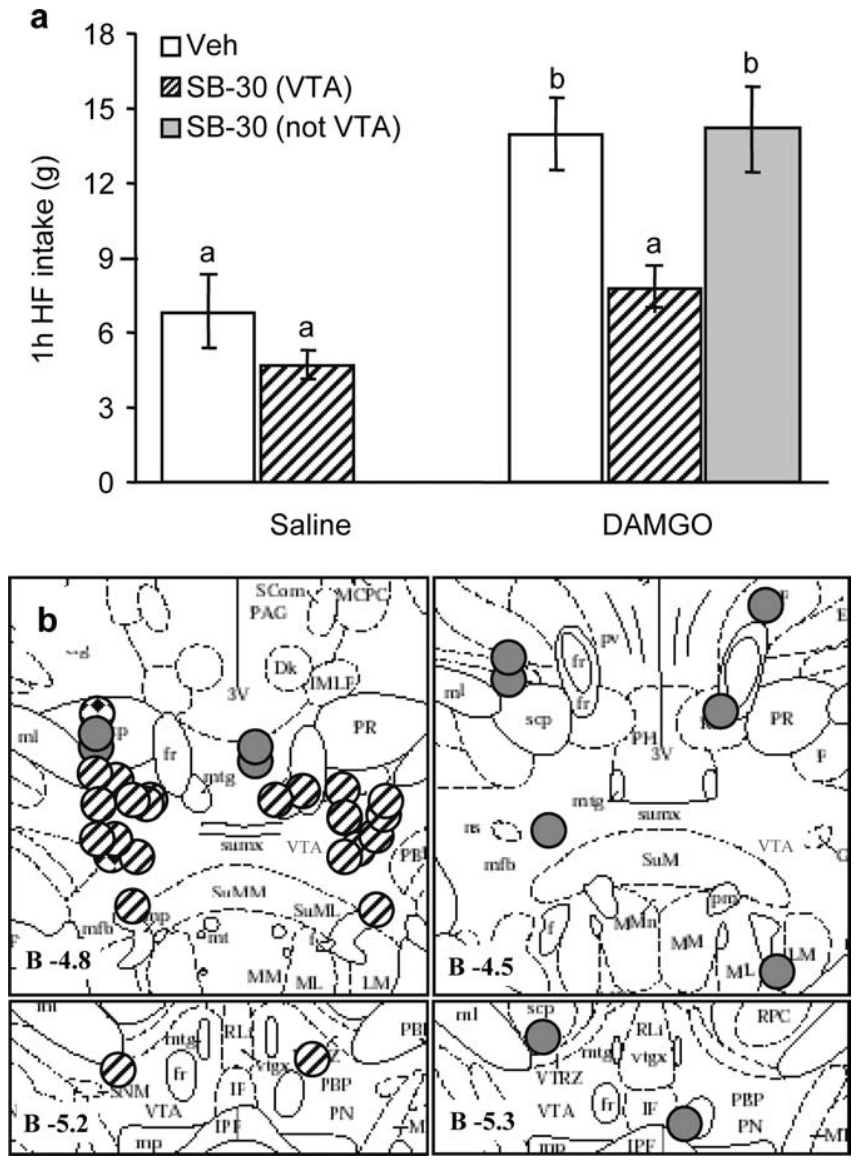

Figure 3. Orexin-1 receptor antagonist administration into the VTA blocks high-fat intake induced by accumbens administration of DAMG0.A, Vehicle (Veh) or the orexin receptor antagonist SB334867 (SB) (15 nmol/side) was injected into the VTA and saline or DAMG0 (250 ng) into the nucleus accumbens after overnight access to high-fat (HF) chow for presatiation. The robust DAMG0-induced feeding response over saline baseline was almost completely abolished by VTA pretreatment with the orexin receptor antagonist (NS). In animals with either one or both of the bilateral cannula tips not within the VTA, the orexin receptor antagonist was unable to block DAMGO-induced high-fat feeding. Bars that do not share the same letter are significantly $(p<0.05)$ different from each other, based on ANOVA. $\boldsymbol{B}$, Verification of orexin receptor antagonist injection sites aimed at the VTA. Striped circles depict animals with both sites within the VTA $(n=11)$, gray circles depict animals with one or both sites outside the VTA $(n=6)$, and diamond-filled circles depict animals with unilateral injections $(n=2)$. Injection sites are superimposed on images from the Paxinos and Watson (1997) stereotaxic atlas.

Double immunohistochemistry for Fos and orexin revealed many double-labeled neurons in this sector of the hypothalamus (Fig. 6 ). Whereas $\sim 35 \%$ of orexin neurons spontaneously coexpressed c-Fos after accumbens saline injection, $80 \%$ of orexin neurons coexpressed c-Fos after accumbens DAMGO injection in the dorsomedial area $\left(F_{(1,5)}=34.9 ; p=0.002\right)$ (Fig. 7). In the area dorsolateral to the fornix, there was no significant DAMGOinduced activation of orexin neurons $\left(F_{(1,5)}=1.72 ; p=0.25\right)$.

In the area dorsomedial to the fornix, a majority (55\%) of neurons expressing c-Fos after accumbens DAMGO were orexin positive, but in other areas of the hypothalamus, including the area dorsolateral and ventral to the fornix, a majority of nonorexin neurons were activated by accumbens DAMGO. Because preliminary observations showed that very few melaninconcentrating hormone-IR neurons exhibited DAMGO-induced Fos expression (data not shown), the neurochemical phenotype of these non-orexin neurons remains to be identified. 

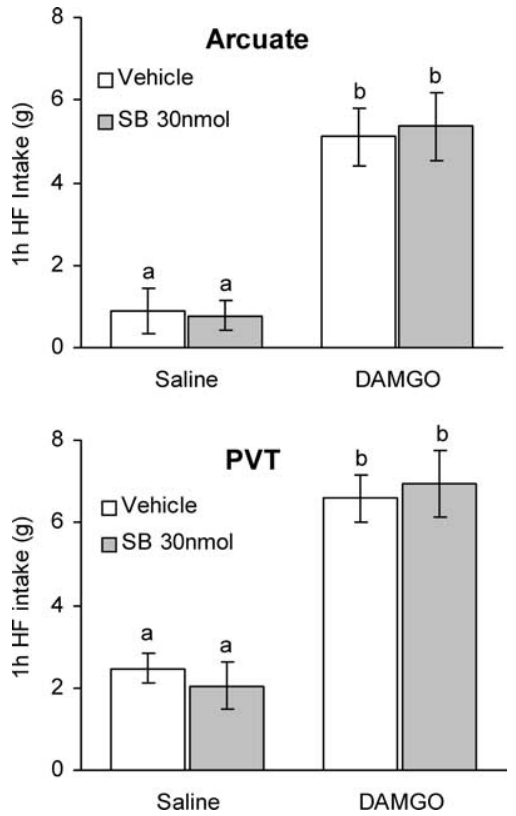

Figure 4. Orexin-1 receptor antagonist administration into the arcuate nucleus or PVT does not affect high-fat (HF) intake induced by accumbens DAMGO. Vehicle or the orexin receptor antagonist SB334867 (SB) (15 nmol/side) was injected into the arcuate nucleus of the hypothalamus (top) or the PVT (bottom) and saline or DAMGO $(250 \mathrm{ng})$ into the nucleus accumbens immediately after the $1 \mathrm{~h}$ presatiation period with high-fat chow. In contrast to the VTA, orexin receptor antagonist administration into the arcuate nucleus and PVT did not change DAMGOinduced high-fat intake. Bars that do not share the same letter are significantly $(p<0.01)$ different from each other, based on ANOVA.

Accumbens sites yielding DAMGO-induced eating project to orexin neurons in the perifornical hypothalamus

To examine the presence of direct projections from DAMGOresponsive accumbens injection sites to hypothalamic orexin neurons, we injected the anterograde tracer BDA mixed with DAMGO in the same injection volume. Inspection of hypothalamic sections from a rat that exhibited significant DAMGOinduced fat intake revealed the presence of many varicose axon profiles in the perifornical area closely associated with doublestained orexin-positive neurons (Fig. 6). The projections appeared to travel mainly through the medial forebrain bundle, which contained many heavily labeled short axon profiles. From this bundle, axons penetrated medially to innervate the lateral hypothalamus, including the perifornical area, and parts of the dorsomedial and paraventricular hypothalamic nuclei (data not shown). Unilateral accumbens injections resulted in staining that was confined almost entirely to the ipsilateral side, with only the stray axon profile on the contralateral side.

\section{Discussion}

Using two independent models, the present findings clearly demonstrate a role for orexin signaling in accumbens-induced highfat appetite. This conclusion is further supported by functional anatomical data showing that accumbens DAMGO administration increases Fos expression in a set of orexin neurons and that projections originating in the nucleus accumbens terminate in close proximity to hypothalamic orexin neurons.

The behavioral results confirm previous reports that manipulation of the nucleus accumbens with the $\mu$-opioid agonist DAMGO induces voracious appetite for high-fat food in satiated rats (Zhang et al., 1998; Zhang and Kelley, 2000; Kelley et al., 2002; Will et al., 2003). Our presatiation paradigm with the same
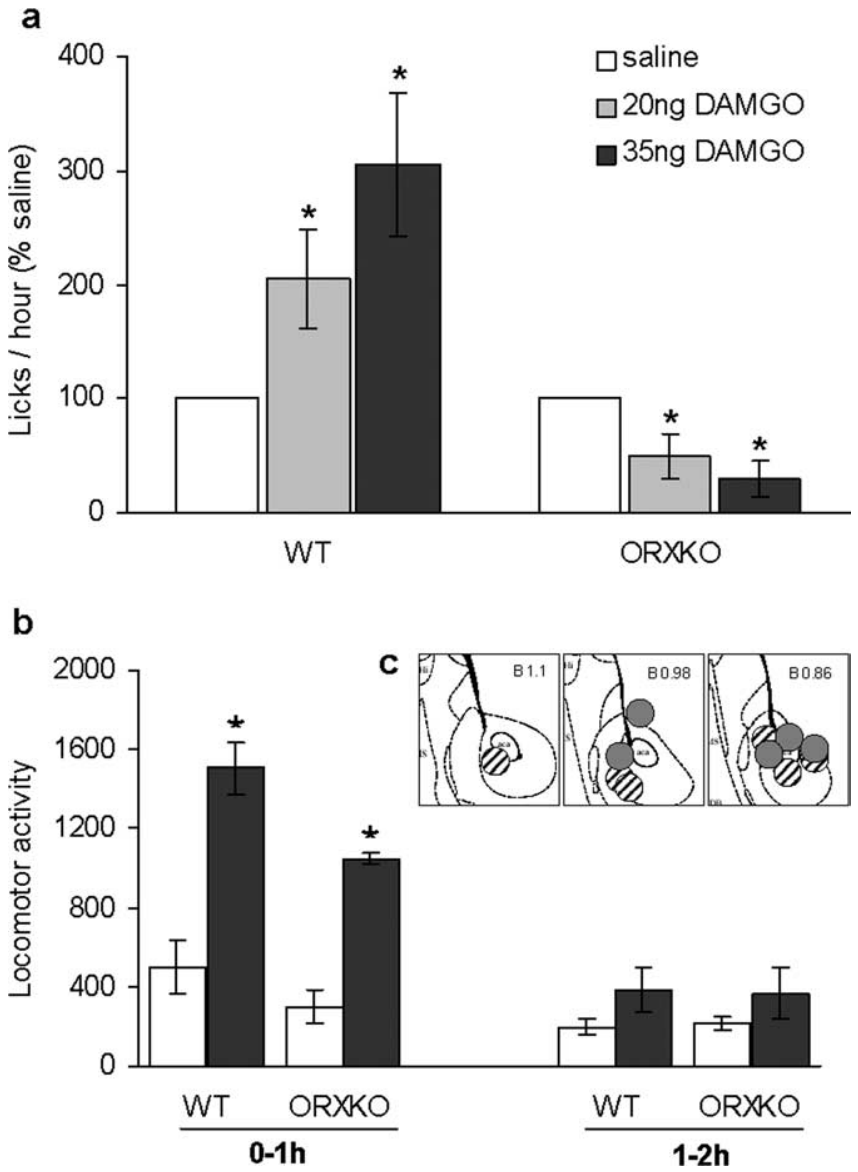

Figure 5. Orexin-deficient mice do not increase high-fat intake after intra-accumbens injection of DAMG0. $\boldsymbol{a}$, DAMG0 ( 20 or $35 \mathrm{ng}$ ) administered to the nucleus accumbens significantly increases intake of $20 \%$ corn oil in wild-type (WT) $(n=5)$ but not in orexin knock-out (ORXKO) $(n=6)$ mice during the second hour of access. * indicates significantly different compared with saline control. $\boldsymbol{b}$, DAMGO $(35 \mathrm{ng}$ ) administered unilaterally to the nucleus accumbens increases locomotor activity in both wild-type and orexin-deficient mice during the first but not during the second hour of access to corn oil. c, Injection sites in wild-type (gray circles) and orexin knock-out (striped circles) mice. Injection sites are superimposed on images from the Paxinos and Watson (1997) stereotaxic atlas.

palatable diet emphasizes the fact even more that this appetite occurs despite the presence of very strong satiety signals. Our rats ingested a significant portion of their daily food intake in the hour just before the DAMGO injection. Therefore, their stomachs must have been greatly distended, absorption of nutrients in full progress, and nutrient sensors completely satisfied during the $2 \mathrm{~h}$ period after injections. However, accumbens DAMGO was able to completely override this intense satiety as demonstrated by the ingestion of another $8.2 \mathrm{~g}$ of high-fat diet. Because of its robust and temporally controlled effect, we used accumbens DAMGO-induced high-fat eating as an experimental model to investigate the downstream neural mechanisms, because they may be more generally involved in palatability and reward-driven food intake.

We found that activation of the hypothalamic orexin system and particularly its projection to the VTA are critical for the behavioral effect of accumbens DAMGO. We first showed that lateral ventricular pretreatment with the selective OX1R blocker SB334867 attenuated DAMGO-induced high-fat intake by $\sim 70 \%$ and that bilateral injection of the antagonist into the VTA blocked the behavior completely. We did not observe any overt side effects of the blocker, making it unlikely that the suppression 
of food intake was attributable to malaise or other ill effects. Using intraperitoneal administration, it was shown that the profile of food intake suppression is very different for SB334867 and malaise producing lithium chloride, with the antagonist preserving the structure of feeding behavior but selectively enhancing the behavioral satiety sequence (Rodgers et al., 2000; Rodgers et al., 2001; Ishii et al., 2004, 2005). The fact that even the highest dose of ventricular administration did not completely suppress DAMGO-induced feeding suggests that the blocker did not diffuse at high enough concentration to the VTA.

To further test the hypothesis that the hypothalamic orexin system is involved in this pharmacologically evoked behavior, we used the orexin-deficient mouse model. Whereas accumbens DAMGO administration significantly increased corn oil intake from a drinking spout, DAMGO-induced corn oil intake was completely absent in orexin-deficient mice. Together, our experiments in rats and mice strongly suggest that orexin signaling in the VTA is critically involved in the stimulation of palatable food intake with DAMGO injected into the nucleus accumbens.

Involvement of the orexin system in mechanisms of reward and drug abuse has been well documented (for review, see Harris and Aston-Jones, 2006; Fields et al., 2007; Sakurai, 2007). Hypothalamic orexin neurons send axonal projections to the VTA (Peyron et al., 1998), including dopaminergic neurons (Nakamura et al., 2000; Balcita-Pedicino and Sesack, 2007), and both dopaminergic and nondopaminergic neurons in the VTA are excited by orexins (Korotkova et al., 2003; Borgland et al., 2006). Although both orexin receptor subtypes OX1R and OX2R are expressed by VTA (Trivedi et al., 1998; Marcus et al., 2001) and specifically in dopamine neurons (Narita et al., 2006), the functional relevance of the OX1R is better characterized because of the availability of the selective antagonist SB334867.

Specifically, orexin signaling is involved in cocaine- and morphine-induced hyperlocomotion and place preference through the mesolimbic dopamine system, partly by potentiating NMDA-mediated excitatory currents in dopaminergic neurons (Borgland et al., 2006; Narita et al., 2006). Orexindeficient mice are less susceptible to develop drug dependence (Georgescu et al., 2003), and orexin injection into the VTA can reinstate an extinguished preference for drugs of abuse (Harris et al., 2005).

We show here that orexin signaling in the VTA also abolishes the robust high-fat eating response to DAMGO administration into the nucleus accumbens, suggesting that this pathway extends to seeking food reward. Among the many targets of the mesolimbic dopamine system, the nucleus accumbens, prefrontal cortex, amygdala, and LH are the most prominent players in the reward system. Although the VTA-accumbens projections consist of a higher percentage of dopamine neurons than the VTA-PFC projections (for review, see Fields et al., 2007), orexin injection into the VTA preferentially increases dopamine efflux in the prefron- b

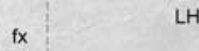

Saline
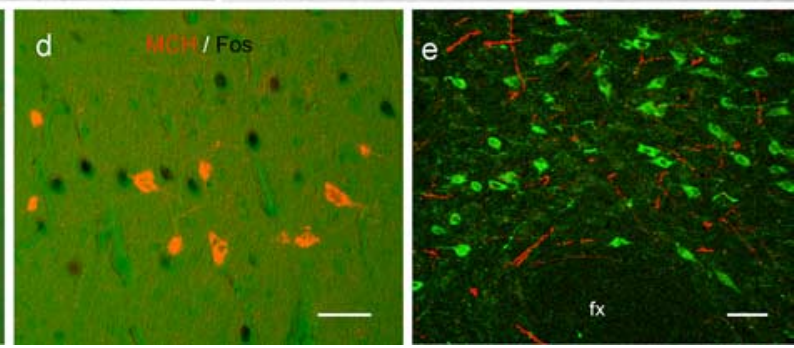

Figure 6. DAMGO induces C-Fos in hypothalamic orexin neurons, and DAMGO-responsive sites project to the hypothalamic pectangular boxes indicate the areas selected for quantitative analysis in Figure 4. c, DAMGO induces

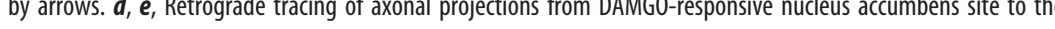
perifornical hypothalamus. Lower- and higher-magnification images of BDA-labeled axon profiles (red) in close anatomica (green) in perifornical area of hypothalamus. fx, Fornix. Scale bars: $\boldsymbol{a}, \boldsymbol{b}, 200 \mu \mathrm{m} ; \boldsymbol{c}-\boldsymbol{e}, 50 \mu \mathrm{m}$.

tal cortex (Vittoz and Berridge, 2006). In addition to dopamine neurons, there is also a significant population of GABA neurons in the VTA with projections mainly to targets other than the nucleus accumbens that are involved in positive reinforcement (Fields et al., 2007). Thus, $\mu$-opioid action in the nucleus accumbens is in a position to modulate activity in the PFC and other key brain areas involved in both the "liking" and "wanting" of palatable food (Berridge, 2007).

Involvement of orexin signaling in the VTA does, however, not preclude other sites of orexin signaling and/or signaling via other peptide and transmitter systems to participate in stimulation of high-fat intake by DAMGO. GABA $\mathrm{A}_{\mathrm{A}}$ agonist-induced neuronal inhibition produced by local administration of the $\mathrm{GABA}_{\mathrm{A}}$ agonist muscimol not only in the VTA but also the nucleus of the solitary tract blocks the ability of DAMGO to stimulate high-fat feeding (Will et al., 2003). Hypothalamic neurons expressing $\mathrm{MCH}$ could also be involved, because they are known to project back to the nucleus accumbens (Georgescu et al., 2005). We focused our initial analysis on orexin because very few $\mathrm{MCH}$ neurons expressed c-Fos after DAMGO administration to the nucleus accumbens.

DAMGO more than doubled the proportion of orexin neurons expressing c-Fos compared with saline control injection in an area dorsomedial but not lateral to the fornix. This suggests that only a specific population of orexin neurons is important for DAMGO-induced intake of high-fat diet. A dichotomy in orexin neuron function has been proposed recently based on the observation that drug- and food-associated conditioned cues increased the percentage of Fos-activated orexin neurons only in the lateral hypothalamus but not in the perifornical and dorsomedial hypothalamus (Harris and Aston-Jones, 2006). Together with the finding that the diurnal change in the percentage of Fos-activated orexin neurons is only seen in the perifornical and medial/dorsomedial hypothalamus (Estabrooke et al., 2001), it appears that, whereas orexin neurons in the lateral hypothalamus are impli- 

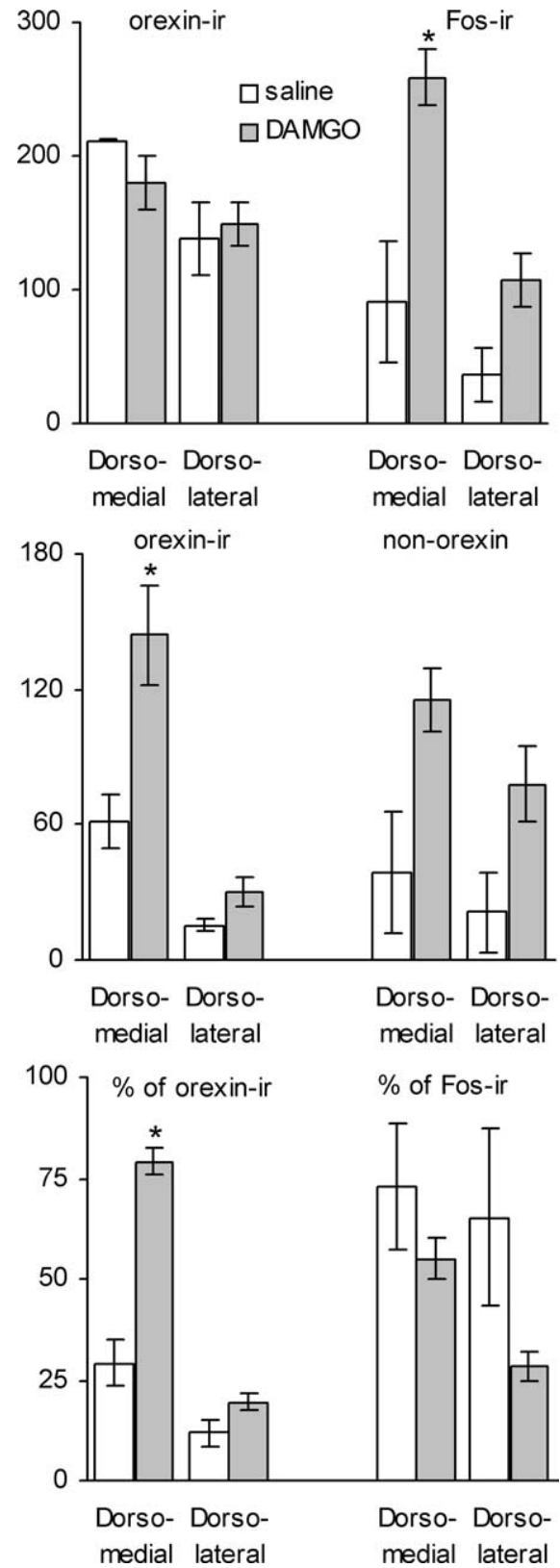

Figure 7. DAMG0 significantly increases the percentage of Fos-expressing orexin neurons in selective areas of the hypothalamus. Top, Numbers of neurons expressing c-Fos after intraaccumbens DAMGO or saline injection and orexin-immunoreactive neurons in two areas of the hypothalamus (see Fig. 6). Middle, Numbers of Fos-IR neurons expressing orexin immunoreactivity (double-labeled) and non-orexin neurons expressing Fos immunoreactivity. Bottom, Double-labeled neurons expressed as percentage of orexin-IR and Fos-IR neurons. Note that the number and percentage of orexin-IR neurons expressing DAMG0-induced c-Fos (doublelabeled neurons) is significantly higher compared with saline $\left({ }^{*} p<0.05\right)$ only in the area dorsomedial to the fornix.

cated in reward, orexin neurons in the perifornical and dorsomedial hypothalamus are implicated in arousal (Harris and AstonJones, 2006). According to this dichotomy, activation of orexin neurons in the more medial hypothalamus as observed in our study would suggest that accumbens DAMGO was arousing rather than rewarding. Although there is no doubt that the DAMGO-injected rats were aroused when they were voraciously eating, the ability of local injection of orexin receptor antagonist into the VTA to block this behavior strongly suggests that the reward pathway was also simultaneously activated. Clearly, orexin neurons in the perifornical hypothalamus have projections to the VTA (Fadel and Deutch, 2002; Geisler and Zahm, 2005).

Our behavioral experiments do not provide information regarding the neural pathway(s) involved in DAMGOinduced activation of orexin neurons. Significant direct projections from the nucleus accumbens to the hypothalamus have been demonstrated. As shown with various tracers and methods, these projections originate mainly from the shell and terminate predominantly in the lateral and perifornical hypothalamus (Conrad and Pfaff, 1976; Groenewegen and Russchen, 1984; Usuda et al., 1998; Otake and Nakamura, 2000). Our results with anterograde tracing in a limited number of rats with cannula placements yielding robust DAMGOinduced intake of high-fat diet clearly show labeled axon profiles in close anatomical proximity to orexin-immunoreactive neurons in the lateral, perifornical, and parts of the medial hypothalamus. This is in contrast to a recent study in transgenic mice expressing green fluorescent protein in mediumspiny striatal output neurons (Sano and Yokoi, 2007). It was found that these neurons terminate in a distinct region of the anterior LH containing mainly glutamate-expressing neurons and few orexin or $\mathrm{MCH}$ neurons. Our tracing results may be different because we used rats and because we injected the tracer in a larger volume to mimic the DAMGO injections. Also, because GABA is an inhibitory transmitter, it is clear that GABAergic accumbens output neurons cannot directly activate hypothalamic orexin neurons and that disinhibition of excitatory inputs are involved. Thus, it is likely that local hypothalamic glutamatergic neurons are causing orexin neuron activation (Sano and Yokoi, 2007) as indicated by the expression of c-Fos.

In conclusion, the present results strongly implicate projections from the nucleus accumbens to parts of the hypothalamic orexin field, activation of orexin neurons, and downstream signaling through the orexin-1 receptor in the VTA, in accumbens-driven intake of palatable foods. It remains to be determined whether this pathway is important for natural food reward.

\section{References}

Backberg M, Hervieu G, Wilson S, Meister B (2002) Orexin receptor-1 (OXR1) immunoreactivity in chemically identified neurons of the hypothalamus: focus on orexin targets involved in control of food and water intake. Eur J Neurosci 15:315-328.

Balcita-Pedicino JJ, Sesack SR (2007) Orexin axons in the rat ventral tegmental area synapse infrequently onto dopamine and gammaaminobutyric acid neurons. J Comp Neurol 503:668-684.

Balthasar N, Dalgaard LT, Lee CE, Yu J, Funahashi H, Williams T, Ferreira M, Tang V, McGovern RA, Kenny CD, Christiansen LM, Edelstein E, Choi B, Boss O, Aschkenasi C, Zhang CY, Mountjoy K, Kishi T, Elmquist JK, Lowell BB (2005) Divergence of melanocortin pathways in the control of food intake and energy expenditure. Cell 123:493-505.

Berridge KC (1996) Food reward: brain substrates of wanting and liking. Neurosci Biobehav Rev 20:1-25.

Berridge KC (2007) The debate over dopamine's role in reward: the case for incentive salience. Psychopharmacology (Berl) 191:391-431.

Berthoud HR (2004) Mind versus metabolism in the control of food intake and energy balance. Physiol Behav 81:781-793.

Borgland SL, Taha SA, Sarti F, Fields HL, Bonci A (2006) Orexin A in the VTA is critical for the induction of synaptic plasticity and behavioral sensitization to cocaine. Neuron 49:589-601.

Clegg DJ, Air EL, Woods SC, Seeley RJ (2002) Eating elicited by orexin-a, but not melanin-concentrating hormone, is opioid mediated. Endocrinology 143:2995-3000.

Conrad LC, Pfaff DW (1976) Autoradiographic tracing of nucleus accumbens efferents in the rat. Brain Res 113:589-596. 
Date Y, Ueta Y, Yamashita H, Yamaguchi H, Matsukura S, Kangawa K, Sakurai T, Yanagisawa M, Nakazato M (1999) Orexins, orexigenic hypothalamic peptides, interact with autonomic, neuroendocrine and neuroregulatory systems. Proc Natl Acad Sci USA 96:748-753.

Estabrooke IV, McCarthy MT, Ko E, Chou TC, Chemelli RM, Yanagisawa M, Saper CB, Scammell TE (2001) Fos expression in orexin neurons varies with behavioral state. J Neurosci 21:1656-1662.

Fadel J, Deutch AY (2002) Anatomical substrates of orexin-dopamine interactions: lateral hypothalamic projections to the ventral tegmental area. Neuroscience 111:379-387.

Fields HL, Hjelmstad GO, Margolis EB, Nicola SM (2007) Ventral tegmental area neurons in learned appetitive behavior and positive reinforcement. Annu Rev Neurosci 30:289-316.

Geisler S, Zahm DS (2005) Afferents of the ventral tegmental area in the rat-anatomical substratum for integrative functions. J Comp Neurol 490:270-294.

Georgescu D, Zachariou V, Barrot M, Mieda M, Willie JT, Eisch AJ, Yanagisawa M, Nestler EJ, DiLeone RJ (2003) Involvement of the lateral hypothalamic peptide orexin in morphine dependence and withdrawal. J Neurosci 23:3106-3111.

Georgescu D, Sears RM, Hommel JD, Barrot M, Bolanos CA, Marsh DJ, Bednarek MA, Bibb JA, Maratos-Flier E, Nestler EJ, DiLeone RJ (2005) The hypothalamic neuropeptide melanin-concentrating hormone acts in the nucleus accumbens to modulate feeding behavior and forced-swim performance. J Neurosci 25:2933-2940.

Groenewegen HJ, Russchen FT (1984) Organization of the efferent projections of the nucleus accumbens to pallidal, hypothalamic, and mesencephalic structures: a tracing and immunohistochemical study in the cat. J Comp Neurol 223:347-367.

Guan JL, Saotome T, Wang QP, Funahashi H, Hori T, Tanaka S, Shioda S (2001) Orexinergic innervation of POMC-containing neurons in the rat arcuate nucleus. NeuroReport 12:547-551.

Harris GC, Aston-Jones G (2006) Arousal and reward: a dichotomy in orexin function. Trends Neurosci 29:571-577.

Harris GC, Wimmer M, Aston-Jones G (2005) A role for lateral hypothalamic orexin neurons in reward seeking. Nature 437:556-559.

Ishii Y, Blundell JE, Halford JC, Upton N, Porter R, Johns A, Rodgers RJ (2004) Differential effects of the selective orexin-1 receptor antagonist SB-334867 and lithium chloride on the behavioural satiety sequence in rats. Physiol Behav 81:129-140.

Ishii Y, Blundell JE, Halford JC, Upton N, Porter R, Johns A, Rodgers RJ (2005) Satiety enhancement by selective orexin-1 receptor antagonist SB-334867: influence of test context and profile comparison with CCK8S. Behav Brain Res 160:11-24.

Kelley AE (2004) Ventral striatal control of appetitive motivation: role in ingestive behavior and reward-related learning. Neurosci Biobehav Rev 27:765-776.

Kelley AE, Berridge KC (2002) The neuroscience of natural rewards: relevance to addictive drugs. J Neurosci 22:3306-3311.

Kelley AE, Bakshi VP, Haber SN, Steininger TL, Will MJ, Zhang M (2002) Opioid modulation of taste hedonics within the ventral striatum. Physiol Behav 76:365-377.

Kelley AE, Baldo BA, Pratt WE (2005) A proposed hypothalamic-thalamicstriatal axis for the integration of energy balance, arousal, and food reward. J Comp Neurol 493:72-85.

Korotkova TM, Sergeeva OA, Eriksson KS, Haas HL, Brown RE (2003) Excitation of ventral tegmental area dopaminergic and nondopaminergic neurons by orexins/hypocretins. J Neurosci 23:7-11.

Marcus JN, Aschkenasi CJ, Lee CE, Chemelli RM, Saper CB, Yanagisawa M, Elmquist JK (2001) Differential expression of orexin receptors 1 and 2 in the rat brain. J Comp Neurol 435:6-25.

Mogenson GJ, Jones DL, Yim CY (1980) From motivation to action: functional interface between the limbic system and the motor system. Prog Neurobiol 14:69-97.

Muroya S, Funahashi H, Yamanaka A, Kohno D, Uramura K, Nambu T, Shibahara M, Kuramochi M, Takigawa M, Yanagisawa M, Sakurai T, Shioda S, Yada T (2004) Orexins (hypocretins) directly interact with neuropeptide $\mathrm{Y}, \mathrm{POMC}$ and glucose-responsive neurons to regulate $\mathrm{Ca}^{2+}$ signaling in a reciprocal manner to leptin: orexigenic neuronal pathways in the mediobasal hypothalamus. Eur J Neurosci 19:1524-1534.
Nakamura T, Uramura K, Nambu T, Yada T, Goto K, Yanagisawa M, Sakurai T (2000) Orexin-induced hyperlocomotion and stereotypy are mediated by the dopaminergic system. Brain Res 873:181-187.

Narita M, Nagumo Y, Hashimoto S, Narita M, Khotib J, Miyatake M, Sakurai T, Yanagisawa M, Nakamachi T, Shioda S, Suzuki T (2006) Direct involvement of orexinergic systems in the activation of the mesolimbic dopamine pathway and related behaviors induced by morphine. J Neurosci 26:398-405.

Otake K, Nakamura Y (2000) Possible pathways through which neurons of the shell of the nucleus accumbens influence the outflow of the core of the nucleus accumbens. Brain Dev 22 [Suppl 1]:S17-S26.

Parsons MP, Li S, Kirouac GJ (2006) The paraventricular nucleus of the thalamus as an interface between the orexin and CART peptides and the shell of the nucleus accumbens. Synapse 59:480-490.

Paxinos G, Watson C (1997) The rat brain in stereotaxic coordinates, Ed 3. San Diego: Academic.

Pecina S, Berridge KC (2005) Hedonic hot spot in nucleus accumbens shell: where do $\mu$-opioids cause increased hedonic impact of sweetness? J Neurosci 25:11777-11786.

Peyron C, Tighe DK, van den Pol AN, de Lecea L, Heller HC, Sutcliffe JG, Kilduff TS (1998) Neurons containing hypocretin (orexin) project to multiple neuronal systems. J Neurosci 18:9996-10015.

Rodgers RJ, Halford JC, Nunes de Souza RL, Canto de Souza AL, Piper DC, Arch JR, Blundell JE (2000) Dose-response effects of orexin-A on food intake and the behavioural satiety sequence in rats. Regul Pept 96:71-84.

Rodgers RJ, Halford JC, Nunes de Souza RL, Canto de Souza AL, Piper DC, Arch JR, Upton N, Porter RA, Johns A, Blundell JE (2001) SB-334867, a selective orexin-1 receptor antagonist, enhances behavioural satiety and blocks the hyperphagic effect of orexin-A in rats. Eur J Neurosci 13:1444-1452.

Sakurai T (2007) The neural circuit of orexin (hypocretin): maintaining sleep and wakefulness. Nat Rev Neurosci 8:171-181.

Sano H, Yokoi M (2007) Striatal medium spiny neurons terminate in a distinct region in the lateral hypothalamic area and do not directly innervate orexin/hypocretin- or melanin-concentrating hormone-containing neurons. J Neurosci 27:6948-6955.

Smith KS, Berridge KC (2005) The ventral pallidum and hedonic reward: neurochemical maps of sucrose "liking" and food intake. J Neurosci 25:8637-8649.

Stratford TR, Kelley AE (1997) GABA in the nucleus accumbens shell participates in the central regulation of feeding behavior. J Neurosci 17:4434-4440.

Stratford TR, Kelley AE (1999) Evidence of a functional relationship between the nucleus accumbens shell and lateral hypothalamus subserving the control of feeding behavior. J Neurosci 19:11040-11048.

Trivedi P, Yu H, MacNeil DJ, Van der Ploeg LH, Guan XM (1998) Distribution of orexin receptor mRNA in the rat brain. FEBS Lett 438:71-75.

Usuda I, Tanaka K, Chiba T (1998) Efferent projections of the nucleus accumbens in the rat with special reference to subdivision of the nucleus: biotinylated dextran amine study. Brain Res 797:73-93.

Vittoz NM, Berridge CW (2006) Hypocretin/orexin selectively increases dopamine efflux within the prefrontal cortex: involvement of the ventral tegmental area. Neuropsychopharmacology 31:384-395.

Volkow ND, Wise RA (2005) How can drug addiction help us understand obesity? Nat Neurosci 8:555-560.

Will MJ, Franzblau EB, Kelley AE (2003) Nucleus accumbens $\mu$-opioids regulate intake of a high-fat diet via activation of a distributed brain network. J Neurosci 23:2882-2888.

Zhang M, Kelley AE (2000) Enhanced intake of high-fat food following striatal mu-opioid stimulation: microinjection mapping and fos expression. Neuroscience 99:267-277.

Zhang M, Gosnell BA, Kelley AE (1998) Intake of high-fat food is selectively enhanced by mu opioid receptor stimulation within the nucleus accumbens. J Pharmacol Exp Ther 285:908-914.

Zheng H, Corkern M, Stoyanova I, Patterson LM, Tian R, Berthoud HR (2003) Peptides that regulate food intake: appetite-inducing accumbens manipulation activates hypothalamic orexin neurons and inhibits POMC neurons. Am J Physiol Regul Integr Comp Physiol 284:R1436-R1444. 